\title{
ASPECTOS BÁSICOS DE LA EDUCACIÓN DE MAYORES: UNA EXPERIENCIA DE FORMACIÓN
}

\author{
BASIC NOTIONS OF EDUCATION FOR THE ELDERLY: A TRAINING EXPERIENCE
}

\section{NOÇÕES BÁSICAS DE EDUCAÇÃO PARA ADULTOS IDOSOS: UMA EXPERIÊNCIA DE FORMAÇÃO}

María José Víquez Barrantes*

\begin{abstract}
Resumen: A continuación se presenta el relato de la experiencia desarrollada en el marco del proyecto ED- 3053 "Formación para personas facilitadoras de procesos de enseñanza-aprendizaje con personas adultas y adultas mayores”, del Instituto de Investigaciones en Educación (INIE) en coordinación con el Programa Institucional para la Persona Adulta y Adulta Mayor (PIAM) de la Universidad de Costa Rica. El objetivo fundamental del proyecto es desarrollar un curso titulado "Aspectos Básicos de la Educación para mayores” para personas facilitadoras de los cursos del PIAM, específicamente mediante tres grandes temas: I. Aspectos básicos de gerontología, II. Procesos de enseñanza aprendizaje para mayores y III. Técnicas didácticas para la educación de mayores. Durante el 2014 se realizaron dos experiencias en las cuales, participó un total de 30 personas facilitadoras que imparten cursos de artesanías, idiomas, computación, actividad física, arte y cultura y estilos de vida saludables en el PIAM. La experiencia educativa del curso, brindó a los participantes, herramientas teóricas y prácticas para mejorar los procesos de enseñanza-aprendizaje con población adulta mayor, así como brindó una serie de contenidos conceptuales, procedimentales y actitudinales para desarrollar procesos educativos con personas mayores. Además, con la experiencia se legitima la labor educativa que persigue el Programa y se logran hacer rupturas con ese imaginario negativo que señala que las personas mayores "ya no pueden aprender"
\end{abstract}

Palabras clave: Educación. Personas adultas mayores. Formación para facilitadores. Gerontología. PIAM.

Abstract: This paper presents the experience developed in the project ED-3053 "Training for facilitators of teaching and learning process with adults and elderly people" - Institute for Research in Education (INIE) in coordination with the Institutional Program for Older Adults (PIAM) of the University of Costa Rica. The main objective of the project is to develop a course entitled "Basic Education for the Elderly" for the facilitators of the PIAM courses, specifically through three main themes: I. Basic concepts of Gerontology, II. The processes of teaching and learning for the elderly and III. Didactic techniques for education of the elderly. During 2014, two experiments were carried out, involving a total of 30 facilitators who teach courses in handicrafts, languages, computers, physical activity, art and culture and healthy lifestyles at PIAM. The educational experience of the course provided to the participants theoretical and practical tools to improve teaching and learning of the elderly population and also a number of conceptual, procedural and attitudinal educational processes to work with the

\footnotetext{
* Assistente Social e Mestranda de Gerontologia pela Universidade da Costa Rica. Diploma em Gestão da Administração Gerontological pela Pontificia Universidade Católica de Chile. Professora do Departamento do Serviço Social da Universidade da Costa Rica. Assessora de Educação da Programa Institucional para Adultos Maiores (PIAM) da Universidade da Costa Rica. E-mail: mariajose.viquez@ucr.ac.cr
} 
elderly. In addition, with the experience, the educational work followed by the Program is legitimized and it is possible to break up the negative imaginary that indicates that older people "cannot learn."

Keywords: Education. Elderly. Training for facilitators - PIAM Gerontology.

Resumo: Este texto apresenta a experiência desenvolvida no projeto ED-3053 "Formação para facilitadores de processo de ensino e aprendizagem com pessoas adultas e idosas” - Instituto de Investigaçóes em Educação (INIE) em coordenação com o Programa Institucional para Adultos Mayores (PIAM) da Universidade da Costa Rica. O principal objetivo do projeto é desenvolver um curso intitulado "Noçoes básicas de educação para adultos maiores" para os facilitadores dos cursos PIAM, especificamente através de três temas principais: I. Noções básicas de Gerontologia, II. Os processos de ensino e de aprendizagem para idosos e III. Técnicas didáticas para educação de pessoas idosas. Durante o ano de 2014, foram realizadas duas experiências, nas quais foram envolvidos um total de 30 facilitadores que ensinan cursos de artesanato, idiomas, informática, atividade física, arte e cultura e estilos de vida saudáveis no PIAM. A experiência educativa do curso proporcionou aos participantes ferramentas teóricas e práticas para melhorar o ensino-aprendizagem da população idosa, e forneceu um número de processos educativos conceituais, procedimentais e atitudinais para desenvolver com pessoas idosas. Além disso, com a experiência legitima-se o trabalho educacional perseguido pelo Programa e é possível fazer rupturas no imaginário negativo que indica que as pessoas mais velhas "não podem aprender”.

Palavras-chave: Educação. Pessoas idosas. Formação para facilitadores. Gerontologia-PIAM.

\section{Introducción}

A mediados de los años ochenta, en la Universidad de Costa Rica, se gesta un proyecto impulsado por el Dr. Alfonso Trejos Willis para incluir a las personas mayores de 50 años de edad en los cursos regulares impartidos por las diferentes carreras; esto motivado en parte por la creación de las “Universidades de la Tercera Edad” (UTE) en Francia. Desde entonces el Programa Institucional para la Persona Adulta y Adulta Mayor (PIAM) ha procurado responder a las necesidades de este grupo mediante la inclusión y la participación social en los diversos espacios que proporciona la Universidad de Costa Rica.

El PIAM se enmarca en la educación permanente (Murillo y Rapso, 2005), una orientación que concibe a la educación como un proceso que dura toda la vida, donde las personas adquieren, acumulan conocimientos y habilidades en la cotidianidad. La educación permanente plantea entonces, que no hay una etapa para estudiar y otra para actuar; que aprender y desaprender es un proceso que se construye a partir de la experiencia y a través de toda la vida.

En este sentido, la institución universitaria ha debido adaptar los contenidos, las metodologías de enseñanza, las técnicas didácticas y, fundamentalmente, la propia filosofía del proceso de enseñanzaaprendizaje a las expectativas, necesidades e intereses de las personas mayores; pues estas instituciones se concibieron como formadoras de recurso humano para el campo profesional y la inclusión en el mercado laboral.

La ruptura en las formas tradicionales de educación y la apertura hacia nuevas maneras de interacción que involucren el aprendizaje, es un reto para los programas universitarios que tiene la doble responsabilidad de brindar oportunidades educativas y de capacitar al personal que las facilite. 
En 2011 se inicia en el PIAM un proceso que pretende capacitar a las personas facilitadoras de los diferentes cursos específicos, y en 2014 se consolida esta iniciativa con el curso "Aspectos básicos de la educación para mayores”, para enriquecer sus conocimientos y su desempeño en los procesos educativos, a partir del conocimiento de aspectos básicos de gerontología, procesos de enseñanza aprendizaje para mayores y técnicas didácticas para la educación de mayores.

Durante el 2014 se realizaron dos experiencias en las cuales, participó un total de 30 personas facilitadoras que imparten cursos de artesanías, idiomas, computación, actividad física, arte y cultura y estilos de vida saludables. Este equipo de facilitadores está formado por personas con gran expertiz en sus respectivas áreas, pues basan su accionar en la experiencia, sin embargo, no todas cuentan con instrucción básica (o formal) en relación con la gerontología, la didáctica, ni los procesos de enseñanza-aprendizaje para personas adultas y adultas mayores.

La presente ponencia se propone rescatar las experiencias de dicho proceso de capacitación para identificar los aportes realizados a los procesos de enseñanzaaprendizaje que llevan a cabo los y las facilitadoras participantes del curso.

\section{Objetivos}

El objetivo general del proyecto consiste en capacitar desde una perspectiva gerontológica, a las personas facilitadoras de los cursos del PIAM, en los temas de aspectos básicos de gerontología, procesos de enseñanza aprendizaje para mayores y técnicas didácticas para la educación de mayores, con el fin de enriquecer sus conocimientos y su desempeño en los procesos educativos. Como objetivo específico se desarrolló un curso de capacitación semestral para abordar en tres módulos los aspectos básicos de gerontología, los procesos de enseñanza aprendizaje para mayores y las técnicas didácticas para la educación de mayores.

\section{Metodología}

El curso propone dieciséis (16) lecciones por semestre, una vez a la semana de 31/2 horas. El módulo I comprende el abordaje de temas como Envejecimiento y vejez: principales conceptos - Mi propio proceso de envejecimiento - Cambios biopsicosociales que ocurren en la vejez, entre otros. El módulo II desarrolla tópicos como El perfil de la persona facilitadora de procesos de enseñanza aprendizaje para mayores- ¿Qué es la educación para mayores? - ¿Cómo aprenden los mayores?, entre otros. En el módulo III se tratan aspectos como El manejo de los grupos - Recursos y materiales didácticosDinámicas para el trabajo con mayores, así como algunos aspectos relacionados con la evaluación. El curso combina exposiciones magistrales, trabajos grupales y prácticas individuales para desarrollar los diferentes temas de manera presencial.

RESULTADOS OBTENIDOS: La experiencia educativa del curso "Aspectos básicos para la educación de mayores” generó una serie de aportes significativos en la población participante. Algunos de los más importantes fueron:

\section{- Aportes del curso “Aspectos básicos de la educación para mayores":}

Por aportes se entiende aquellas contribuciones desde los contenidos conceptuales, procedimentales y actitudinales, proporcionados en el curso “Aspectos básicos 
de la educación para mayores” a las personas participantes.

En este sentido, el curso estuvo orientado no solamente al saber como tal (aprender conocimientos, disponer de información), sino que incorpora otros saberes igual de importantes, que es el saber hacer (capacitarse en procedimientos y en formas de actuar) y querer ser, es decir aquellas actitudes y valores que permiten tener una predisposición mejor ante la realidad.

Estos saberes se reflejan en los tipos de contenidos que incluye el curso, los cuales se dividen en contenidos conceptuales, procedimentales y actitudinales; con el fin de buscar un mayor análisis de cada uno, sin embargo es importante señalar que están sumamente interrelacionados.

A continuación ahondaré en cada uno de los aportes que se propiciaron a partir del curso:

\section{- A nivel de contenidos conceptuales:}

A nivel de contenidos conceptuales, el curso desarrolló 3 módulos temáticos orientados a la nivelación de conocimientos básicos sobre la educación para personas adultas mayores. De los subtemas tratados algunos generaron mayor interés entre las personas participantes, por ejemplo, los cambios biosicosociales que ocurren en el proceso de envejecimiento, pues su definición, explicación y ejemplos permitieron comprender que son paulatinos, que forman parte de un proceso natural de los seres humanos, que hay factores que los aceleran y que no necesariamente son negativos, pues hay cambios de desarrollo y crecimiento.

Otro de los temas que aportó conceptualmente fue la definición de los programas universitarios para mayores y los objetivos educativos que sustentan su existencia, en este sentido, varias de las personas participantes manifestaron haber entendido por primera vez de qué se trataba el PIAM, y por qué era importante implementar los contenidos de los programas de cada uno de los cursos por encima de la necesidad de socialización que puedan manifestar algunos estudiantes.

Por otro lado, los aportes del curso fueron evidentes cuando se facilitaron los temas: conceptos relevantes de la gerontología educativa y qué es la educación para mayores; ambos temas fueron identificados como el enlace entre lo teórico y metodológico en el curso y se pudo constatar en la revisión que se hizo de los programas de los cursos, pues lograron articular los diferentes contenidos para hacer una crítica constructiva, informada y con propiedad de las propuestas de otros compañeros, ejercicio que posteriormente aplicaron a sus propios programas. Cabe mencionar que el avance en cuanto a la capacidad de análisis crítico de los temas fue evidente con el transcurrir de las sesiones, de manera que con frecuencia se les reconoció el esfuerzo realizado y la participación en cada clase.

Consultados sobre los aportes en este ámbito, las personas participantes afirmaron que el curso les permitió: conocer estadísticas actualizadas sobre la situación de las personas adultas mayores y proyecciones demográficas a nivel nacional e internacional; repasar y corregir conceptos como vejez, envejecimiento, andragogía, pedagogía, y conocer algunas de las aproximaciones teórica detrás de los prejuicios y estereotipos hacia la población mayor. 


\section{- A nivel de contenidos procedimentales:}

A partir de la experiencia vivida en el curso, se puede señalar que un aspecto importante para los y las facilitadoras participantes del mismo, no solamente fue conocer de manera conceptual la información referente a la educación para mayores, tal y como lo vimos en el apartado anterior, sino cómo esos conceptos son aplicables al contexto del PIAM.

Por ejemplo, como parte de los contenidos procedimentales que se desarrollaron en el curso, se puede señalar las “Dinámicas de los procesos grupales”, en donde se identificó el concepto de dinámica grupal, los tipos de grupos y los roles que se pueden presentar en cada uno de ellos. Este tema brinda aportes entre los cuales se destaca la importancia de la comunicación, las diferencias entre los miembros de un grupo, el descubrimiento de fortalezas, la interacción entre los miembros y el papel de los líderes.

Este tema también sirve para destacar la importancia de tener en claro la tarea en común que tiene un grupo, por qué y cómo es que funcionan los miembros dentro de un grupo, la importancia de que el facilitador se ocupe de los que no están participando de un grupo cuestionándose el por qué.

Por otro lado se menciona que las personas entre sus necesidades poseen la necesidad de afecto, confirmación y pertenencia, que si son tomadas en cuenta dentro del espacio de clase puede resultar provechoso para la tarea del grupo, ya que logra motivar a los y las estudiantes. En este sentido también es importante que la tarea sea realista, ya que si es muy fácil o genera frustración puede ocasionar que las personas se distraigan de la misma.
Por otro lado, como parte de los contenidos procedimentales, el tema de las metodologías desde la gerontología educativa resultó ser un aporte fundamental para las personas participantes, ya que les brindó herramientas concretas de cómo elaborar una metodología idónea a cada contenido de la clase. Lo anterior queda demostrado, en el programa del curso Cultura del vino, de la siguiente manera:

Método: Logocéntrico: Activo reproductivo: Para aprender de la cultura del vino se debe actuar, practicar y entrenar el proceso de cata con el fin de despertar los sentidos en el análisis organoléptico del vino, razón por la cual esta metodología se considera apta para cumplir debidamente los objetivos del curso. (Programa del curso Cultura del vino II ciclo 2014)

También se refleja en la metodología propuesta en el curso Plantas medicinales I:

Por la naturaleza del curso, en algunos momentos se hará uso del método expositivo- suscitador-estimulador, donde el estudiante participa activamente de acuerdo a sus experiencias previas. También se hará uso del método activoproductivo en el que cada estudiante expone al grupo sobre las prioridades curativas de una planta seleccionada por el o ella. Finalmente cuando el programa así lo amerite el profesor hará uso del método expositivo. (Programa del curso Plantas medicinales I, II ciclo 2014)

Lo anterior nos evidencia que se logra incorporar el tema de las metodologías que se pueden utilizar desde la gerontología educativa y además que se utiliza un argumento adecuado.

Otro de los temas que brindó aportes importantes de manera procedimental fue el conocer cómo utilizar la técnica del portafolio 
como una técnica para evaluar los procesos de enseñanza-aprendizaje con población adulta mayor. A partir de la explicación teórica de la técnica, los y las facilitadoras pudieron aprender a elaborarla de manera creativa. Esto se evidenció en la actividad realizada en la clase, pero principalmente al incorporarse como una estrategia de evaluación en tres de los cursos que se están impartiendo en el II ciclo lectivo 2014.

Uno de ellos fue el curso de Plantas Medicinales I, en el cual se contempló como parte de la rúbrica de evaluación y tiene un valor del 30\% de la nota para pasar al nivel II. Otro ejemplo, es el curso de Bordado en Cintas, en el cual, las estudiantes están elaborando un portafolio con cada una de las técnicas aprendidas en el curso. Además, se incorporó en el curso de Cultura del vino, en el cual se enfatiza que mediante esta técnica los y las estudiantes aprenderán haciendo.

Por otra parte, siguiendo con los aportes de los contenidos procedimentales a los procesos educativos podemos mencionar la consolidación de un programa de curso con un eje gerontológico. Todos los contenidos del curso no sirven de mucho de manera aislada y si no se implementan o aplican en un proyecto; por este motivo, los participantes incorporaron los diferentes contenidos para mejorar los programas de sus respectivos cursos.

Esto lo visualizamos en la justificación del curso "Aspectos fundamentales para el uso de medicamentos” el cual plantea que

Un curso básico sobre medicamentos busca estrechar la relación entre los pacientes adultos y adultos mayores con la terapia farmacológica con el fin de favorecer un uso racional de los medicamentos y mejorar la calidad de vida de los participantes. (Programa del curso
Aspectos fundamentales para el uso de medicamentos, II ciclo 2014)

Donde se contempla las particularidades de la población que participa de los cursos, pero al mismo tiempo se retoman temas acordes a los cambios biosicosociales ocurridos durante el proceso de envejecimiento.

\section{- A nivel de contenidos actitudinales:}

Uno de los aportes más importantes producto de la participación en este curso, considera los contenidos actitudinales, es decir, aquellos que pretenden una motivación diferente y una reflexión interna que genere cambios en los comportamiento y actitudes; ya que no tiene mucho sentido que las personas facilitadoras adquieran una serie de conocimientos referentes a la educación de mayores, aprendan sobre cómo desarrollar procesos educativos para esta población, pero que sigan reproduciendo ideas negativas hacia esta población.

En este sentido, el curso permitió una valoración del propio proceso de envejecimiento para cada una de las personas participantes. Si partimos del hecho de que el envejecimiento es un proceso que dura toda la vida, a todas las personas nos debería de interesar el tema y no solamente aquellas que se encuentran en la etapa de la vejez. Y aunque esto esté claro en la teoría, en la práctica o en la vida cotidiana no se asume de manera tan sencilla.

Como parte de la experiencia, logramos identificar que algunos facilitadores o facilitadoras, no habían reflexionado sobre cómo estaban viviendo su envejecimiento y cómo se estaban preparando para la vejez en el caso de las personas más jóvenes. Por ejemplo, una facilitadora nos señalaba que 
ella nunca se había puesto a reflexionar sobre este tema y se percató que no estaba haciendo ninguna acción en concreto para tener un estilo de vida saludable que posibilite una vejez más satisfactoria; además evidenciamos cómo un facilitador que tenía más de 60 tenía serios problemas en aceptar su propia vejez e incluso la de los demás, como una forma de evadir y negar la realidad.

Lo anterior se vincula con el reconocimiento de los mitos y estereotipos que presentan las personas facilitadoras hacia la población adulta mayor. Se considera que el problema no es tener mitos porque son ideas generalizadas que se transmiten por los diferentes medios de socialización, la dificultad recae justo en no identificar cuáles mitos o estereotipos tenemos y en reproducirlos de manera consciente en los distintos espacios que se comparte con la población, especialmente en los grupos con los cuales se realizan los procesos educativos.

Otro aspecto importante a tomar en cuenta como parte de los aportes actitudinales, fue el reconocimiento de los objetivos educativos del Programa y esto es clave para gestionar un cambio más a nivel cualitativo en el PIAM. La mayoría de las personas facilitadoras consideraban que el Programa no era en esencia educativo, sino recreativo, donde los y las estudiantes vienen a disfrutar de los cursos y a ocupar su tiempo libre, situación más acorde a las motivaciones de participación en un club social y no a los objetivos de un programa educativo.

Esto se refleja en algunas de las expresiones de los y las facilitadoras, tales como: “Ah ya entiendo de qué se trata el Programa" "Yo pensaba que era solamente para que los viejitos pudieran pasar un rato agradable y salir de las casas”. Estas concepciones del Programa no coinciden con su quehacer y funcionamiento, por lo que este tema aportó a la identificación de objetivos y a la búsqueda de la educación como característica fundamental en este tipo de programas.

Esta desvinculación entre los objetivos del PIAM y el pensamiento que tienen los y las facilitadoras del mismo, nos dice que aún tenemos muchos retos y uno de ellos es el generar en las personas facilitadoras un sentido de pertenencia al programa, aspecto que se logra mejorar con la participación en este curso, ya que se forma una identidad de grupo al contar con un espacio de intercambio entre ellos. Esto fue uno de los aportes más significativos para los y las participantes del curso, ya que lograron conocerse entre sí, sentirse parte del programa y acercarse de manera más concreta al personal que labora en el PIAM.

Esto permitió darle una mayor legitimidad social y funcional al curso, ya que no solamente adquirió importancia por los contenidos que se abordaron, sino por los vínculos y dinámicas de los procesos grupales que se generaron.

\section{- Cambios vividos en la experiencia:}

El curso se desarrolló con el temario propuesto, sin embargo se realizaron algunos cambios en la metodología programada, respondiendo sobre todo a las necesidades de información e intereses de las personas participantes.

En este sentido, por ejemplo se incorporó el tema de resolución de conflictos en el aula, y se profundizó en los procesos de memoria y aprendizaje de las personas adultas mayores. Ambos temas fueron planteados por los participantes como fundamentales para comprender mejor las dinámicas que se desarrollan en el aula, principalmente el primero de éstos pues con frecuencia deben 
mediar en los conflictos entre estudiantes o hacer frente a situaciones que los involucra directamente. Manifestaron sentirse más seguros y en sintonía con el Programa para la próxima vez que enfrenten algún problema.

Además, se realizaron cambios en algunas de las guías de trabajo de la sesión, esto con el fin de promover la participación de las personas facilitadoras, de mantener su interés y motivarles a compartir sus experiencias. Estas guías de trabajo se establecían con antelación para orientar la sesión, distribuir el tiempo dedicado a las actividades y dejar las asignaciones respectivas, sin embargo, en el desarrollo de la sesión podía modificarse lo propuesto de acuerdo a la dinámica y nivel de participación del grupo.

Otro ejemplo de cambios vividos en la experiencia del curso, es el recorte del número de sesiones establecidas inicialmente por un acuerdo del grupo, ya que varios de los participantes se ausentarían en las últimas sesiones, así que se acordó juntar un par de temas para no afectar mayormente el curso. $\mathrm{Al}$ ser tan participativas las sesiones esto no acarreó ningún inconveniente en el avance de los temas, pues con la lecturas asignadas y las discusiones en clase su logró cumplir con lo propuesto.

\section{- Contradicciones presentes en la experiencia:}

El trabajo con personas y las experiencias de esta naturaleza son esencialmente complejas y llenas de contradicciones.

Una de las contradicciones que experimentamos las facilitadoras fue el hecho de realizar un gran esfuerzo en impartir el curso y la sobrecarga laboral que esto generó. Ambas responsables del proyecto hemos asumido el compromiso de planear el proyecto, de negociarlo ante el Instituto de Investigaciones en Educación, de ejecutarlo, preparando las clases diarias del mismo y de organizar la logística; es decir, no solamente somos encargadas de la parte académica del proyecto sino también de lo administrativo, teniendo poca ayuda operativa del mismo Programa. Esta situación no valida la propuesta en sí y desmotiva la experiencia como tal, entonces queda la duda de que si este proyecto es de tanto interés para el PIAM, ¿por qué no se le brinda el apoyo necesario?

Esto genera dudas en la sostenibilidad del mismo proyecto, ya que si no genera el interés suficiente para que se le brinde el soporte necesario, es difícil que se mantenga a lo largo del tiempo. Aunado a que los compromisos adquiridos en este curso, fue el asumir la formación facilitadores/as como un espacio permanente; es decir, que al PIAM se le está exigiendo la capacitación continua en temas asociados a la educación de mayores.

Otra de las contradicciones que se presentaron a lo largo del curso, se refiere al debate de conceptos utilizados para definir lo que es la educación de mayores, principalmente la cuestión entre Andragogía versus Gerontología Educativa. Esta contradicción académica, se desarrolló porque una de las invitadas especiales entendía la educación de mayores como andragogía, cuando las facilitadoras del curso la comprenden como gerontología educativa. Esta situación, aunque en principio generó dudas en los/as participantes, motivó a discutir los conceptos y enriqueció la propuesta como tal.

\section{- Concepción de educación}

La concepción de educación que predominó a lo largo del curso, se toma principalmente de los aportes de la educación 
popular, en la cual, no concibe que la educación sea tradicional, bancaria y que las personas participantes sean pasivas; todo lo contrario, se parte de la idea de la construcción del conocimiento y que el proceso de enseñanzaaprendizaje es horizontal y dialéctico, es decir enseñamos aprendiendo y aprendemos enseñando.

Paulo Freire señala en su libro "Pedagogía de la autonomía: saberes necesarios para la práctica educativa” una serie de principios sobre el proceso de enseñanza-aprendizaje, necesarios a tomar en cuenta. Nos interesa destacar los siguientes:

Enseñar no es transferir conocimiento: Tal y como se mencionó, partimos de la idea de que el conocimiento se construye, y en el caso del curso lo pudimos constatar de buena manera, ya que las facilitadoras del curso aportamos las herramientas teóricas en materia de educación para mayores, pero las personas participantes aportaron todo un bagaje de conocimientos aplicados ya en la práctica, es decir, ellos/as ya tienen una experiencia fundamental a la hora de impartir clases en el PIAM.

Enseñar exige la aprehensión de la realidad: Freire habla de la capacidad de aprender del ser humano y nos indica que

“la capacidad de aprender no solo para adaptarnos sino sobre todo para transformar la realidad, para intervenir en ella y recrearla, habla de nuestra educabilidad en un nivel distinto del nivel de adiestramiento de los otros animales o del cultivo de las plantas” (FREIRE, 1997, p.32)

Esto se liga con otro principio que nos indica que Enseñar exige la convicción de que el cambio es posible, en este sentido, en el curso fue fundamental partir de estos saberes, ya que como Programa nos interesa que las personas facilitadoras puedan transformar las prácticas educativas que viven cotidianamente, no solamente en beneficio de ellos/as sino a favor de las personas adultas mayores que participan de las mismas.

Enseñar exige alegría y esperanza: Y esto fue una característica que predominó a lo largo del curso. Se pudieron desarrollar metodologías participativas aplicadas al contexto PIAM y lo más importante es que el espacio siempre contó con un clima alegre y esperanzador.

Todo lo anterior, nos exige como Programa, seguir promoviendo este tipo de espacios y nos da el reto de apostar por la educación permanente como una forma de movilización, del continuo aprehendizaje y de seguir promoviendo una mejor calidad de vida en la población meta de nuestros servicios.

\section{- Contexto social, cultural, económico:}

La población de Costa Rica continúa el proceso de envejecimiento demográfico. Según el Instituto Nacional de Estadística y Censos, la población de personas adultas de 65 años y más alcanza el 7,3\% del total del país (INEC, 2013).

Lo que llama la atención de este proceso es lo acelerado que se manifiesta, pues para el 2025 se espera que la población mayor supere el 15\%; de manera que el tema del envejecimiento ha logrado un espacio importante en la agenda pública para proponer y discutir proyectos encaminados a dar respuesta a las necesidades de la población.

La educación es uno de los derechos que cobra importancia en esta discusión y en los proyectos de vida de las personas adultas mayores (que cada vez son más longevas), y es la Universidad una de las instancias responsables de garantizar la existencia de 
oportunidades educativas a lo largo de toda la vida.

En este contexto se ubica, desde 1986, el Programa Institucional para la Persona Adulta y Adulta Mayor (PIAM), programa universitario dirigido a personas mayores de 50 años y jubiladas, y que ofrece cupos en cursos regulares y específicos. Los cursos específicos (solo para personas mayores de 50 años) son impartidos en su mayoría por facilitadores voluntarios, especialistas en áreas como el movimiento humano, artesanías, manualidades, arte, idiomas, computación, entre otras.

Como pionero de la educación para mayores en Costa Rica, el PIAM ha tenido que desarrollar procesos de formación y capacitación para sus funcionarias, y tenía pendiente implementarlos para sus facilitadores, necesidad que quedó evidenciada a lo largo del quehacer de varios años. Es en este contexto que se establece y desarrolla el curso, la iniciativa es novedosa a nivel nacional, ya que ninguna otra institución propicia espacios formales de capacitación para esta población y en este tema.

A partir de la experiencia vivida, se puede formular una serie de conclusiones teórico-prácticas y recomendaciones con el objetivo de identificar los principales aprendizajes, mejorar nuestra vivencia y también favorecer otras prácticas educativas de esta naturaleza.

\section{Conclusiones}

A partir de este curso se evidencia la necesidad de un programa de capacitación y actualización constante ofrecido por el PIAM a sus facilitadores y facilitadoras, ya que a lo largo de los talleres surgían comentarios por parte de los mismos de lo "útil y rico" que les resultaba asistir a los talleres. Además, estos espacios permiten fortalecer la unión de los facilitadores y facilitadores, así como su identificación con la institución del PIAM, y compartir experiencias entre ellos y ellas, que les permitan enriquecer sus conocimientos y tener herramientas para actuar ante situaciones emergentes.

La experiencia educativa del curso “Aspectos básicos para la educación de mayores” generó una serie de aportes significativos en la población participante. Algunos de los más importantes fue el brindar herramientas teóricas y prácticas para mejorar los procesos de enseñanza-aprendizaje con población adulta mayor. Brindar una serie de contenidos conceptuales, procedimentales y actitudinales para desarrollar procesos educativos con personas mayores, los cuales se lograron evidenciar en la mejora de los programas de los cursos específicos.

A nivel actitudinal, el curso le permitió a los participantes una reflexión interna sobre el propio proceso de envejecimiento y sobre la reproducción de ideas negativas hacia la población adulta mayor, que repercuten directamente en la implementación de prácticas discriminatorias y experiencias negativas de educación.

En este mismo sentido, reconocer los objetivos educativos que se pretenden en el Programa, permite dar un salto cualitativo, en el quehacer profesional del PIAM, ya que se legitima la labor educativa que persigue el Programa y se logran hacer rupturas con ese imaginario negativo que nos dice que las personas mayores "ya no pueden aprender"

Por otra parte, mediante la implementación de este proyecto, se logra una legitimidad social y funcional del curso, ya que no solamente adquirió importancia por los contenidos que se abordaron, sino por los vínculos y dinámicas de los procesos grupales que se generaron. 
La concepción de educación que predominó a lo largo del curso, no concibe que la educación sea tradicional, bancaria y que las personas participantes sean pasivas; todo lo contrario, se parte de la idea de la construcción del conocimiento y que el proceso de enseñanza-aprendizaje es horizontal y dialéctico, es decir enseñamos aprendiendo y aprendemos enseñando.

El contexto que estamos viviendo en la actualidad, nos respalda la propuesta del curso; ya que la cantidad de personas mayores va en aumento y por ende, más personas están deseosas de incorporarse a estos programas; asimismo, el marco del contexto universitario nos exige programas y servicios que promuevan no tanto la cantidad sino la calidad, tal y como lo perseguimos con este proyecto.

\section{Referências}

FREIRE, P. Pedagogía de la autonomía. Editorial Paz e terra, São Paulo, 1997.

INSTITUTO NACIONAL DE ESTADISTICAS Y CENSOS. Censos Nacionales del año 2011. San José, Costa Rica. Disponível em: http://www.inec.go.cr/

MURILLO, A.; RAPSO, M. Trabajo social: un enfoque gerontológico en programas desarrollados en instituciones de educación superior dirigidos a la población mayor de 50 años. In: Búsquedas del trabajo social latinoamericano: urgencias, propuestas y posibilidades. Editoral Espacio, Buenos Aires. 